\title{
Drenagem percutânea de cisto paraprostático, guiada por ultra-som em um cão
}

\author{
Ultrasound-guided percutaneous drainage of paraprostatic cyst in a dog \\ Neusa Margarida Paulo ${ }^{1}$, Daniel Lessa Mendes ${ }^{2}$, Marcelo Seixo de Brito ${ }^{3}$, \\ Maria da Conceição ${ }^{4}$, Marco Augusto Machado ${ }^{5}$ \& Leandro Guimarães Franco ${ }^{5}$
}

\begin{abstract}
RESUMO
Cistos paraprostáticos no cão ocorrem freqüentemente em animais de raças grandes, idosos e intactos. Eles são preenchidos por líquido amarelo claro a laranja, podendo haver a infecção e abscedação dos mesmos. Esta afecção é relativamente rara quando comparada a outras doenças prostáticas desta espécie. O presente artigo relata a drenagem percutânea ecoguiada de cisto paraprostático em um cão da raça Fila Brasileiro de nove anos de idade. Foi aspirado 620 ml de líquido purulento, de pouca consistência. A cultura e antibiograma do material coletado revelaram a presença de Citrobacter sp., Pseudomonas sp. e Enterobacter sp. O pós-operatório foi feito em casa e por 11 dias o animal estava recuperando-se bem, até o proprietário relatar sua morte súbita, não tendo havido possibilidade de proceder à necropsia. Esta técnica quando realizada de forma adequada é de fácil execução, baixo custo e apresenta resultados satisfatórios, podendo ser utilizada como técnica definitiva ou pré-operatória. Apesar das propriedades positivas da drenagem percutânea serem relatadas na literatura, ressalta-se que o comprometimento do proprietário durante o pós-operatório é fundamental para o sucesso do procedimento.
\end{abstract}

Descritores: cão, próstata, ultra-sonografia, cisto.

\section{ABSTRACT}

In dogs, paraprostate cysts frequently occur in intact animals of larger species and advanced age. Filled by a liquid ranging from light yellow to orange, these cysts may present infection and abscession. This medical condition is relatively rare if compared to other prostate diseases of this type. The present article reports on the echoguided percutaneous drainage of a paraprostate cyst in a 9-year-old Brazilian Fila dog. Six hundred and twenty milliliters of not very consistent purulent liquid was aspirated. The culture and the antibiogram of the matter collected revealed the presence of Citrobacter sp., Pseudomonas sp., and Enterobacter sp. Post-surgery procedures were conducted at home. The animal was recovering well for eleven days, but afterwards the owner reported its sudden death, not being possible to proceed with the necropsy at that time. When executed in a proper way, this low-cost technique can be easily carried out, presenting satisfactory results, and it may be used as a definitive or presurgery method. Notwithstanding the positive attributes of percutaneous drainage reported on literature, it is emphasized that the owner's compromise during post-surgery is fundamental to the success of the procedure.

Key words: dog, prostate, ultrasonography, cyst. 


\section{INTRODUÇÃO}

Cistos paraprostáticos no cão ocorrem freqüentemente em animais de raças grandes, idosos e intactos. Eles são preenchidos por líquido amarelo claro a laranja, podendo haver a infecção e abscedação dos mesmos. Esta afecção é rara quando comparada a outras prostatopatias desta espécie. O seu tratamento consiste da drenagem ou da ressecção do cisto. Em casos recorrentes pode-se proceder a prostatectomia $[2,8]$. Ocorrendo recidiva as drenagens devem ser repetidas $[2,7]$.

A drenagem ecoguiada tem sido considerada efetiva para o tratamento de cistos e abscessos localizados em profundidade. Por ser um procedimento minimamente invasivo, rápido e barato, ele é uma alternativa de primeira escolha, evitando a execução de procedimentos cirúrgicos radicais. As complicações desta manobra são raras e sua eficácia já foi comprovada tanto para o homem quanto para animais. As falhas podem ser representadas por moderada hemorragia e vazamento de conteúdo séptico [3-7]. Resultados superiores são obtidos quando são drenadas coleções de cavidade unilocular bem definida [5].

Apesar das qualidades da drenagem ecodirigida, alguns fatores devem ser considerados como determinantes de falhas. Benoist [1] relata que no homem pode ocorrer a formação de abscessos complexos, fístulas intestinais intercomunicantes, peritonite difusa, presença de tecido necrótico. Cavidades pequenas podem dificultar a inserção do dreno bem como facilitar o deslocamento deste do seu ponto de entrada, resultando em drenagem insuficiente [7,9].

\section{RELATO DE CASO}

Um cão da raça Fila Brasileira, de nove anos de idade foi atendido no Hospital Veterinário da Escola de Veterinária da UFG, com histórico de ter sido assistido em uma clínica veterinária da cidade, onde foi feito um exame ultrassonográfico que mostrou um cisto paraprostático. A indicação inicial era a prostatectomia. O exame clínico geral não revelou outras alterações e o proprietário revelou que o cão apresentava dificuldade para defecar. Repetiu-se o exame ultrassonográfico que revelou presença de uma grande estrutura cística, destacando-se do lobo direito da próstata e estendendo-se lateralmente à direita da vesícula urinária, medindo $12,84 \mathrm{~cm}$ de diâmetro em um dos cortes, com formato arredondado e conteúdo hipoecóico (Figura 1). O proprietário foi informado sobre as alternativas de tratamento para o animal e decidiu aceitar a drenagem ecoguiada.

Fez-se uma ampla tricotomia do abdome caudal e induziu-se a anestesia geral com Tiopental sódico. Com o auxílio do transdutor selecionou-se um ponto lateral ao pênis e introduziu-se uma agulha longa, sempre acompanhando a imagem no monitor. Procedeuse o esvaziamento prostático sempre se observando no monitor (Figura 2), até que ocorresse resistência à aspiração, indicando colabamento da parede do cisto. Foi coletado $620 \mathrm{~mL}$ de conteúdo purulento. Neste momento encerrou-se a drenagem e injetou-se cerca de $10 \mathrm{~cm}$ de Enrofloxaxina na cavidade. A antibioticoterapia foi inicialmente instituída sem os testes de sensibilidade. Recomendou-se a administração diária de Amoxiclina. A cultura e antibiograma do material coletado revelou a presença de Citrobacter sp., Pseudomonas sp. e Enterobacter sp. Todos os microrganismos presentes exibiram em comum sensibilidade a Enrofloxacina e a Orbifloxacina.

Nos dias subseqüentes o proprietário foi contactado para dar informações sobre o animal. Até dez dias após ele revelou que o animal se alimentava normalmente e não apresentava mais dificuldade para defecar. Revelou ainda que o animal estava bem e até corria. Ao ser contactado, aos 12 dias após a drenagem para que lembrasse que devia trazer o animal para nova avaliação, ele declarou que o mesmo havia morrido na véspera, tendo apresentado, segundo suas observações, fraqueza e salivação intensa. Não deu outras informações e não trouxe o cadáver para necropsia.

\section{DISCUSSÃO}

A drenagem percutânea de cistos paraprostáticos é citada como sendo de fácil execução e eficaz na maioria dos casos [1-7,9], podendo ser utilizada como procedimento definitivo, ou pré-operatório em pacientes de alto risco até a melhora da condição cirúrgica $[4,5,7]$. O menor custo a torna uma alternativa economicamente viável [7], tal fator deve ser levado em consideração quando o proprietário não pode arcar com os custos do procedimento cirúrgico, inviabilizando assim o tratamento do animal.

O acompanhamento por ultra-sonografia da drenagem permite acompanhar o esvaziamento do cisto e ter certeza da retirada da maior quantidade de material possível, evitando assim o extravasamento 


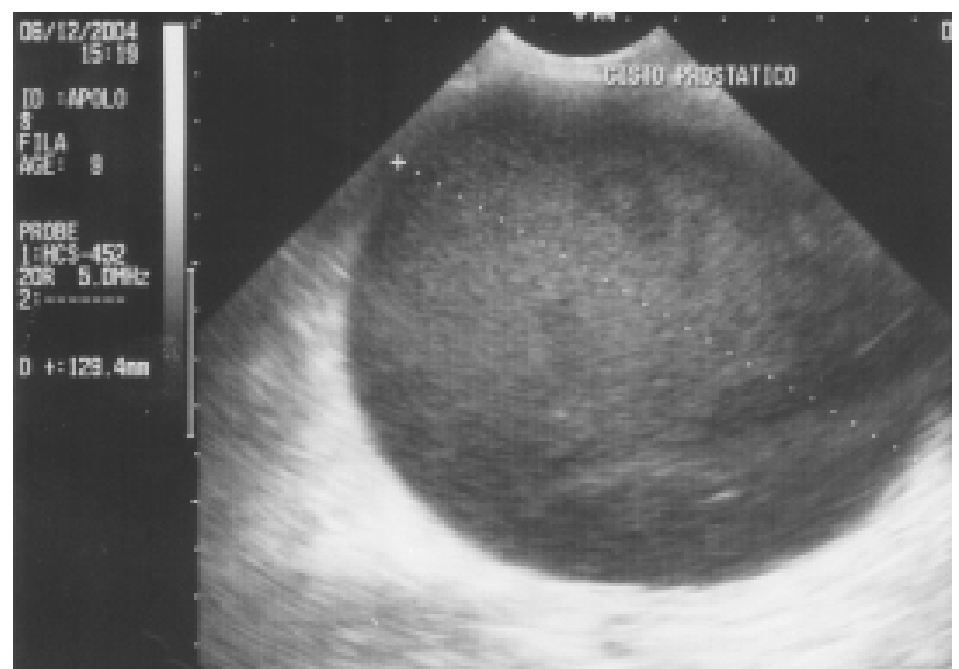

Figura 1. Corte sagital da estrutura cística, antes da drenagem, medindo $12,84 \mathrm{~cm}$ de diâmetro. Utilizado equipamento de ultra-som HONDA HS2000, com transdutor microconvexo de 5,0 MHz.
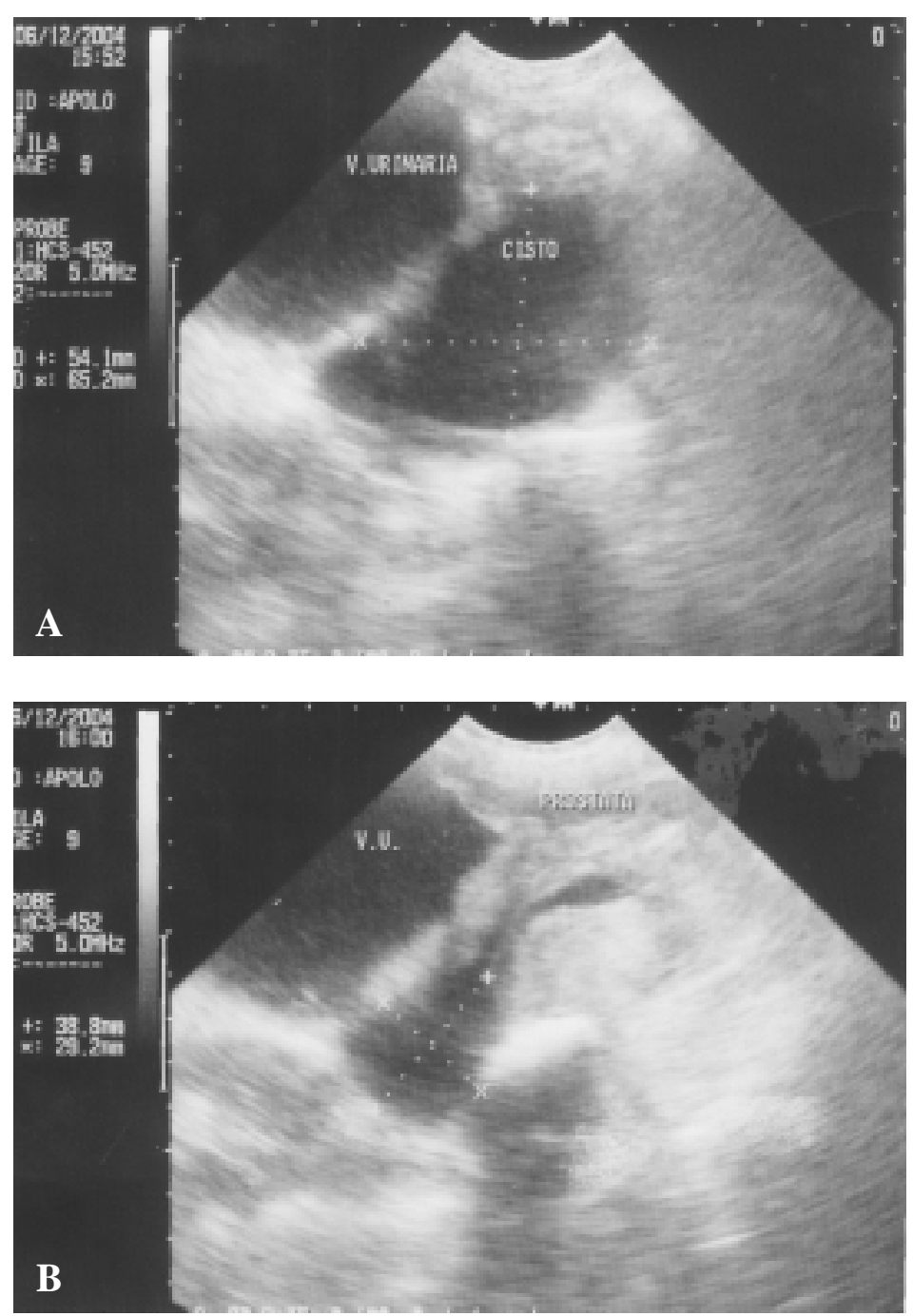

Figura 2 (A e B). Evolução do procedimento de drenagem ecoguiada de cisto prostático. Nota-se o esvaziamento progressivo da coleção purulenta. do material [1,7], o diagnóstico diferencial deve incluir as prostatites, tumores prostáticos e granulomas [6,7], podendo ser realizado por ultrasonografia e é de fundamental importância para a escolha do procedimento a ser adotado.

A antibioticoterapia é feita de forma empírica até a realização de cultura e antibiograma, para confirmação ou não do antibiótico a ser administrado [6,7,9]. Esta deve persistir por no mínimo 6 semanas [7]. Na medicina humana a Escherichia coli é citada como a bactéria mais freqüentemente encontrada em abscessos prostáticos $[4,6]$, fato que concorda com a incidência citada para cães. O uso de antibióticos deve ser sistemático e baseado na cultura e antibiograma. Em geral os exames de cultura revelam a presença de microrganismos e a colaboração do proprietário do animal é considerada imprescindível para o sucesso do tratamento [7].

A terapia antibiótica tem se mostrado eficiente na maioria dos casos, apresentando grande sucesso $[1,2,4,6,7,9]$. Relata-se ainda baixa mortalidade $[1,2,4,6,7,9]$ a qual é causada principalmente por choque séptico [7,9]. A cooperação do proprietário é de fundamental importância para a recuperação do animal, tanto para a administração da antibioticoterapia, como do retorno do animal à clínica para acompanhamento, já que em muitos casos é necessária mais de uma drenagem $[1,6,7,9]$, ou até outro procedimento cirúrgico.

No presente caso a causa do óbito do animal não pode ser investigada, pois o proprietário se recusou a trazê-lo para necropsia, apesar de relatar que um dia antes do óbito o animal estava bem. Por isso é de extrema importância antes da realização de qualquer procedimento clinico ou cirúrgico, o comprometimento do proprietário com o tratamento do animal [7], já que em muitos casos o pós-operatório é feito em casa, e algumas pessoas não têm noção da dificuldade que vão encontrar.

A participação ativa e o compromisso do proprietário no tratamento do animal submetido à drenagem ecoguiada de cisto paraprostático pode ser determinante para o sucesso do procedimento. 


\section{REFERÊNCIAS}

1 Benoist S., Panis Y., Pannegeon V., Soyer P., Watrin T., Boudiaf M. \& P. Valleur. 2002. Can failure of percutaneous drainage of postoperative abdominal abscesses be predicted? The American Journal of Surgery. 184: 148-153.

2 Boland L.E., Hardie R.J., Gregory S.P. \& Lamb C.R. 2003. Ultrasound-guided percutaneous drainage as the primary treatment for prostatic abscesses and cysts in dogs. Journal of American Animal Hospital Association. 39: 151-159.

3 Brunker J. 2004. Prostate. Ultrasound-guidad fine-needle biopsy and aspiration. Procedures Pro Navc clinicians brief [serial online] Jan [cited 2004, dec 23. Available from: URL http://clinicians brief.com/cb/pastissues/2004/docs/0104-pdf.

4 Civardi G., Di Candio G., Giorgio A., Goletti O., Ceragioli T., Filice C., Caremani M. \& Buscarini L. 1998. Ultrasound guided percutaneous drainage of abdominal abscesses in the hands of the clinician: a multicenter Italian study. European Journal of Ultrasound. 8: 91-99.

5 Civardi G., Filice C., Caremani. M., Giorgio A., Vallisa D., Berté R. \& Cavanna L. 1998. Clinical efficacy of ultrasound guided percutaneous drainage of abscesses in patients with leukaemia and lymphoma. European journal of Cancer. 34: 580-583.

6 Collado A., Palou J., Garcia-Penit J., Salvador J., De la Torre P. \& Vicente J. 1999. Ultrasound-guided needle aspiration in prostatic abscess. Adult Urology. 53: 548-552.

7 Froes T.R., Gonzales J.R.M., Kanayama L.M., Jorge R.C \& Iwasaki M. 2003. Ultra-sonografia intervencionista - drenagem percutânea de lesões cavitária e cistos prostáticos em cães. Clínica Veterinária. 47: 34-40.

8 Hedlund C.S. 2002. Cirurgia dos sistemas reprodutivo e genital. In Fossum T. W. (Ed) Cirurgia de pequenos animais. São Paulo: Roca, pp.571-635.

9 Somuncu I., Saglam M., Yagci S., Tahmaz L., Tasar M \& Ors F. 2003. multiloculated prostate abscess treatment with transretal ultrasound guided transretal needle aspiration and lavage with the saline and antibiotic. Clinical Imaging. 27: 251-255. 